St udy on Crosstal k- Free Pol ar i zat i on Spl i tter Wth El I i pt i cal-Hol e Core Ci rcul ar- Hol e Hol ey Fi bers

\begin{tabular}{|l|l|}
\hline 著者 & ZHANG Zej un, TSUJ I Yasuhi de, EGUCH Nasashi \\
\hline $\begin{array}{l}\text { j our nal or } \\
\text { publ i cat i on t i t l e }\end{array}$ & $\begin{array}{l}\text { J our nal of I i ght wave t echnol ogy : a j oi nt } \\
\text { I EEE/OSA publ i cat i on }\end{array}$ \\
\hline vol une & 32 \\
\hline number & 23 \\
\hline page $\mathrm{r}$ ange & 4558 4564 \\
\hline year & 2014 12-01 \\
\hline URL & ht t p: //hdl . handl e. net /10258/3825 \\
\hline
\end{tabular}


St udy on Crosstal k- Free Pol ar i zat i on Spl i tter Wth El I i pt i cal-Hol e Core Ci rcul ar- Hol e Hol ey Fi bers

\begin{tabular}{|l|l|}
\hline 著者 & ZHANG Zej un, TSUJ I Yasuhi de, EGUCH Nasashi \\
\hline $\begin{array}{l}\text { j our nal or } \\
\text { publ i cat i on t i t l e }\end{array}$ & $\begin{array}{l}\text { J our nal of I i ght wave t echnol ogy : a j oi nt } \\
\text { I EEE/OSA publ i cat i on }\end{array}$ \\
\hline vol une & 32 \\
\hline number & 23 \\
\hline page $\mathrm{r}$ ange & 4558 4564 \\
\hline year & 2014 12-01 \\
\hline URL & ht t p: //hdl . handl e. net /10258/3825 \\
\hline
\end{tabular}




\title{
Study on Crosstalk-free Polarization Splitter with Elliptical-Hole Core Circular-Hole Holey Fibers
}

\author{
Zejun ZHANG, Student member, IEEE, Yasuhide TSUJI, Member, IEEE and Masashi EGUCHI, Member, IEEE
}

\begin{abstract}
We proposed a novel polarization splitter based on single-polarized elliptical-hole core circular-hole holey fibers (EC-CHFs). We employed the full-vector finite element beam propagation method (FE-BPM) to demonstrate the polarization splitter which is designed by the large hole EC-CHFs (air filling fraction in core is $\mathbf{3 6 . 7 3 \%}$ ) and small hole EC-CHFs (air filling fraction in core is $\mathbf{4 . 0 8 \%}$ ) can completely split an arbitrarily polarized light beam into two orthogonal polarization states without any crosstalk. In addition, we also calculated the tolerance and wavelength dependence of this kind of polarization splitter.
\end{abstract}

Index Terms-polarization splitter, elliptical-hole core circularhole holey fiber, single polarization property, vector finite element method

\section{INTRODUCTION}

Photonic crystal fibers (PCFs) [1], [2], consisting of single material with a periodical array of air holes around the fiber core, also known as holey fibers (HFs), have attracted a lot of attention in the last two decades due to their flexible structure and the special properties that cannot obtained by the conventional fibers, such as large mode area, endless single mode, high nonlinearity, and so on [2]. One of these properties is the absolutely single polarization transmission and a number of single-polarized PCF structures have been proposed [3][10]. In recent years, elliptical-hole core circular-hole holey fiber (EC-CHF), a novel single-polarized holey fiber with a core consisting of elliptical-holes, has been proposed for achieving the single polarization transmission easily [6], [7]. The EC-CHF can be designed to transmit only the $x$ - or $y$ polarization easily by changing the major axis direction of the elliptical-holes in core region.

Polarization splitter (PS), which can split a light beam into two orthogonal polarization states, is very important component in coherent optical communication system and optical-fiber sensing system. In 2003, a PS based on dualcore PCF has been proposed by Zhang and Yang [11], and an extinction ratio (ER) of $-11 \mathrm{~dB}$ and a bandwidth of $80 \mathrm{~nm}$ can be achieved in the PS which is consisted of dual-core PCF with three kinds of different core sizes. After that, several kinds of PSs based on new structure of PCFs were proposed [12]- [18]. In 2004, Saitoh et al. proposed a PS using three-core PCF, which has an ER better than $-20 \mathrm{~dB}$ and a bandwidth of 37 $\mathrm{nm}$ with a device length of $1.9 \mathrm{~mm}$ [12]. In 2013, Lu et al. proposed a PS based on three-core PCF which has an ER as

Z. Zhang and Y. Tsuji are with the Division of Information and Electronic Engineering, Muroran Institute of Technology, Muroran, 050-8585 Japan. M. Eguchi is with the Department of Photonics System Technology, Chitose Institute of Science and Technology, Chitose 066-8655, Japan.

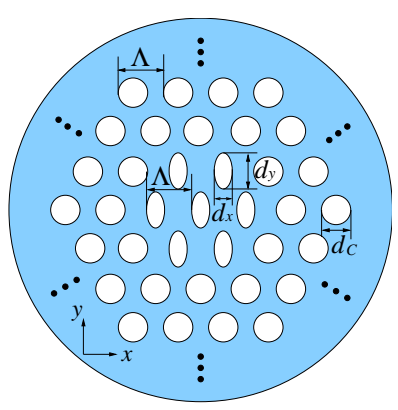

Fig. 1. Elliptical-hole core circular-hole holey fiber (The $y$ EC-CHF).

low as $-20 \mathrm{~dB}$ and a bandwidth of $400 \mathrm{~nm}$ [17]. However, this splitter has long coupling lengths of $x$ - and $y$-polarization modes, and there is a quite large difference between the two kinds of coupling lengths. This makes the design of PS become complicated. Although many new kinds of PSs based on PCF have been proposed, none of them can realize the crosstalkfree separation. From this point, we have proposed a novel PS based on EC-CHFs which can split $x$ - and $y$-polarized waves into two different fibers without any crosstalk with a short device length of $630 \mu \mathrm{m}$ [18]. In our research, the coupling lengths for the two orthogonally polarized waves are almost the same, and this makes the PS easy to design.

In this paper, for the practical application, we discuss the performance and the tolerance of the cross-talk free PS with EC-CHFs in detail. Firstly, in order to avoid the complex analysis of actual waveguide structure mentioned in [18], we discussed the design method for PS by employing the fundamental space-filling mode (FSM) of the elliptical holes in core region. Next, we designed the PS with small hole EC-CHFs to obtain the Gaussian like mode field distribution which can improve the compatibility with the standard singlemode fibers (SMFs). And we noted that the small hole PS designed by FSM has the same high design accuracy as the actual waveguide method. Then, the design accuracy of FSM method versus the air filling fraction of the elliptical holes in core regions has been discussed. In addition, we have also investigated the tolerance and wavelength dependence of the proposed PS in detail. It demonstrated that the PS with small hole EC-CHFs has a larger tolerance and a wider wavelength bandwidth than the PS with large hole EC-CHFs. In this manuscript, the full-vectorial finite element method (FV-FEM) [19] has been used to estimate the modal effective index of the waveguide, and the propagation of incident light has been analyzed by using the full-vector finite element beam propagation method (FE-BPM) [20]. 


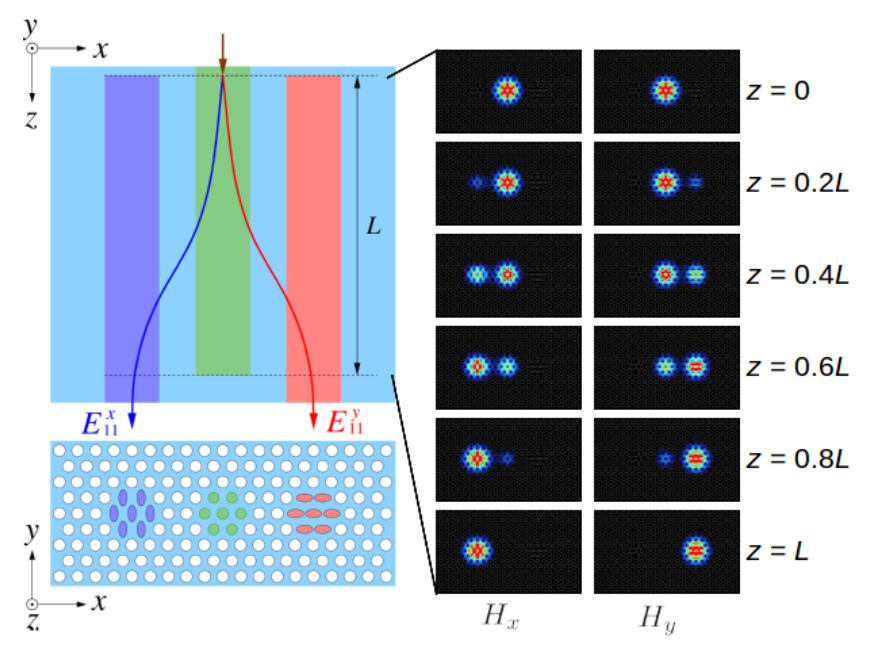

Fig. 2. Structure of proposed PS and the propagation behavior of incident light in the PS by using FE-BPM.

\section{STRUCTURE OF PROPOSED PS}

In this section, we explain the principle of single polarization transmission in the EC-CHF and illustrate the structure of PS with EC-CHFs.

\section{A. PS with EC-CHFs}

As we know, a conventional HF has a sixfold symmetry and has an isotropic in the core region. Birefringence can be obtained by introducing elliptical air holes in the core region and the cross section of an EC-CHF with one ring elliptical hole core is shown in Fig. 1. This kind of EC-CHF is referred to as a $y \mathrm{EC}-\mathrm{CHF}$ due to the major axis of elliptical hole is aligned along the $y$ direction. A $y \mathrm{EC}-\mathrm{CHF}$ can only transmit the $y$-polarized wave, and in the same way, an $x \mathrm{EC}$ CHF, whose major axis of elliptical hole is aligned along the $x$ direction, can only transmit the $x$-polarized wave [7]. In addition, the core region of an EC-CHF is formed by circular hole, whose diameter is smaller than the circular hole in the cladding, has no polarization dependence and both of the $x$ and $y$-polarized waves can be transmitted. Here, we refer to it as a circular-hole core circular-hole holey fiber (CC-CHF).

We have proposed a novel PS which is consisted by three EC-CHFs in parallel as shown in Fig. 2. The waveguide on the left side is a $y \mathrm{EC}-\mathrm{CHF}$, the center is a CC-CHF, and the right side is an $x$ EC-CHF. Using the FE-BPM, we demonstrated that an 45-degree polarized light can be completely divided into two orthogonally polarized waves. The $x$-polarized component $\left(H_{y}\right)$ and $y$-polarized component $\left(H_{x}\right)$ couple only to $x \mathrm{EC}$ $\mathrm{CHF}$ and $y \mathrm{EC}-\mathrm{CHF}$, respectively, as shown in Fig. 2 [18]. This phenomenon can be explained by coupled mode theory.

\section{B. Coupling length and coupling efficiency}

In our proposed PS, the device length $L$ is determined by the average of the coupling lengths of $x$ - and $y$-polarization modes. Using the mode coupling theory, coupling length $L_{c}$ can be calculated by

$$
L_{c}=\frac{0.5 \lambda}{n_{\mathrm{eff}, \mathrm{e}}-n_{\mathrm{eff}, \mathrm{o}}}
$$

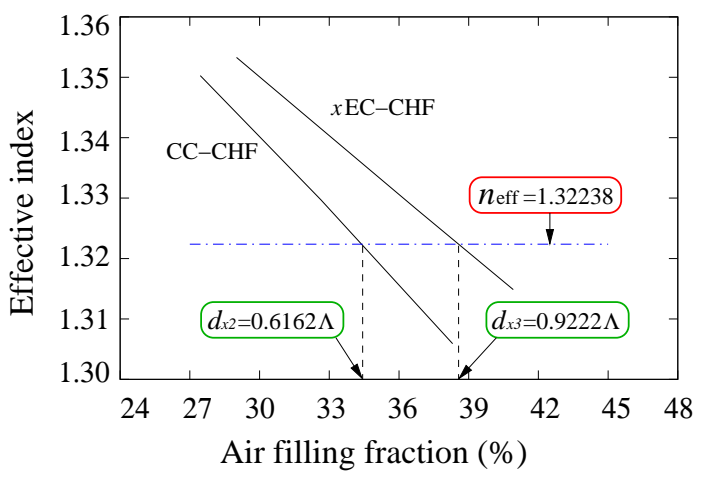

Fig. 3. Effective indices of the FSM in core region for the large hole CC-CHF and $x \mathrm{EC}-\mathrm{CHF}$ as a function of the air filling fraction.

where $\lambda$ is the operation wavelength, $n_{\mathrm{eff}, \mathrm{e}}$ and $n_{\mathrm{eff}, \mathrm{o}}$ are the effective indices of the even and odd modes [18] for $x$-polarization or $y$-polarization. In a coupled system, the coupling efficiency $F$ between the two adjacent waveguides is defined as

$$
F=\frac{\kappa^{2}}{\kappa^{2}+\psi^{2}}=\frac{1}{1+\left(\frac{\psi}{\kappa}\right)^{2}}
$$

with

$$
\psi=\frac{1}{2}\left(\beta_{2}-\beta_{1}\right)=\frac{\pi}{\lambda}\left(n_{\mathrm{eff}, 1}-n_{\mathrm{eff}, 2}\right)
$$

where $\kappa$ is the coupling coefficient between the two adjacent waveguides. $\psi$ is the difference of propagation constants between the two adjacent waveguides in isolated system, and $n_{\mathrm{eff}, 1}$ and $n_{\mathrm{eff}, 2}$ are the respective effective indices of the two waveguides. Using the guide mode in isolated system, coupling length $L_{c}$ can also be defined as follows:

$$
L_{c}=\frac{\pi}{2 \gamma}, \quad \gamma=\sqrt{\kappa^{2}+\psi^{2}}
$$

where $\gamma$ is a quantity which is related to the periodical power transfer between the two adjacent waveguides. When $L_{c}=\pi /(2 \gamma)$, the power of incident light transmits from one waveguide to another completely. From the above, the coupling efficiency $F$ between the two waveguides can be calculated by using the effective indices of the two waveguides in isolated system and the effective indices of the even and odd modes in coupled system, as shown as the following equation.

$$
F=1-\left(\frac{n_{\mathrm{eff}, 1}-n_{\mathrm{eff}, 2}}{n_{\mathrm{eff}, \mathrm{e}}-n_{\mathrm{eff}, \mathrm{o}}}\right)^{2}
$$

In order to design the PS, all the coupled modes and isolated modes are calculated by FV-FEM in this paper.

\section{DESIGN OF PS WITH EC-CHFS}

Here, we present the design examples of our proposed PS with the large hole EC-CHFs (air filling fraction in core is $36.73 \%$ ) and small hole EC-CHFs (air filling fraction in core is $4.08 \%$ ). We employ the FSM of the core region to design the PS efficiently, and the design accuracy of the FSM method is also discussed in detail. 
TABLE I

EFFECTIVE INDICES OF EVEN AND ODD MODES AND THE ESTIMATED COUPLING LENGTHS OF THE PS WITH THE LARGE HOLE EC-CHFS DESIGNED BY FSM

\begin{tabular}{|c|c|c|c|}
\hline \multirow{2}{*}{} & \multicolumn{2}{|c|}{ Effective refractive index } & \multirow{2}{*}{$L_{c}[\mu \mathrm{m}]$} \\
\cline { 2 - 3 } & Even mode & Odd mode & 593 \\
\hline$x$-pol. & 1.310171 & 1.308866 & 592 \\
\hline$y$-pol. & 1.310681 & 1.309073 & 482 \\
\hline
\end{tabular}
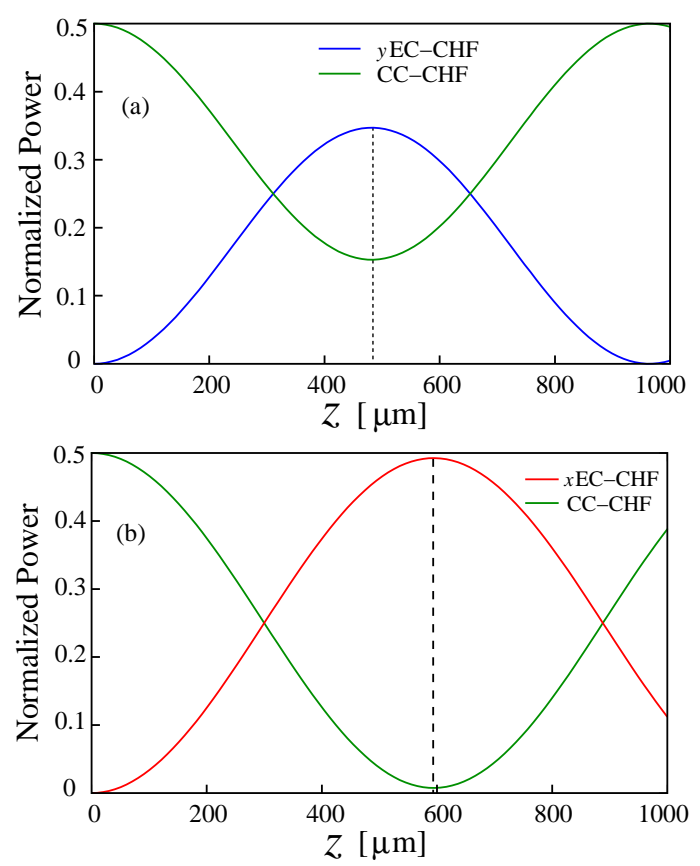

Fig. 4. Normalized power along propagation of (a) $y$-polarized wave, (b) $x$-polarized wave for the PS designed by FSM with the large hole EC-CHFs.

\section{A. Design of PS with large hole EC-CHFs using FSM}

In our research, the PS consisted by three EC-CHFs, and a $y \mathrm{EC}-\mathrm{CHF}$ is set to be the reference waveguide. We design the air hole size in the core region of a CC-CHF and $x \mathrm{EC}-\mathrm{CHF}$ by matching the phase condition. After that, the coupling length between the CC-CHF and $x$ EC-CHF ( $x$-polarization wave) or CC-CHF and $y$ EC-CHF ( $y$-polarization) can be calculated by setting an appropriate separation between the two adjacent cores. Finally, the device length is determined by adopting the average length of the two coupling lengths. Since the coupling lengths of $x$ - and $y$-polarization are independent, each length can be adjusted without any interference to another, so if there is a large difference between the two coupling lengths, we can control each length by adjusting the air hole sizes between the adjacent cores. For example, the coupling length of $y$ polarization will decrease as the hole sizes between the CC$\mathrm{CHF}$ and $y \mathrm{EC}-\mathrm{CHF}$ are reduced.

In [18], we designed the air hole sizes of CC-CHF and $x$ EC$\mathrm{CHF}$ which meet the phase matching condition by the effective index of each waveguide. In this paper, instead to analyze the complicated structure of EC-CHF, we determined the phase matched air holes by using the FSM of the elliptical air holes in core region for each EC-CHF. Here, the parameters of $y$ ECCHF refer to Ref. [18]. The centers of all the air holes are

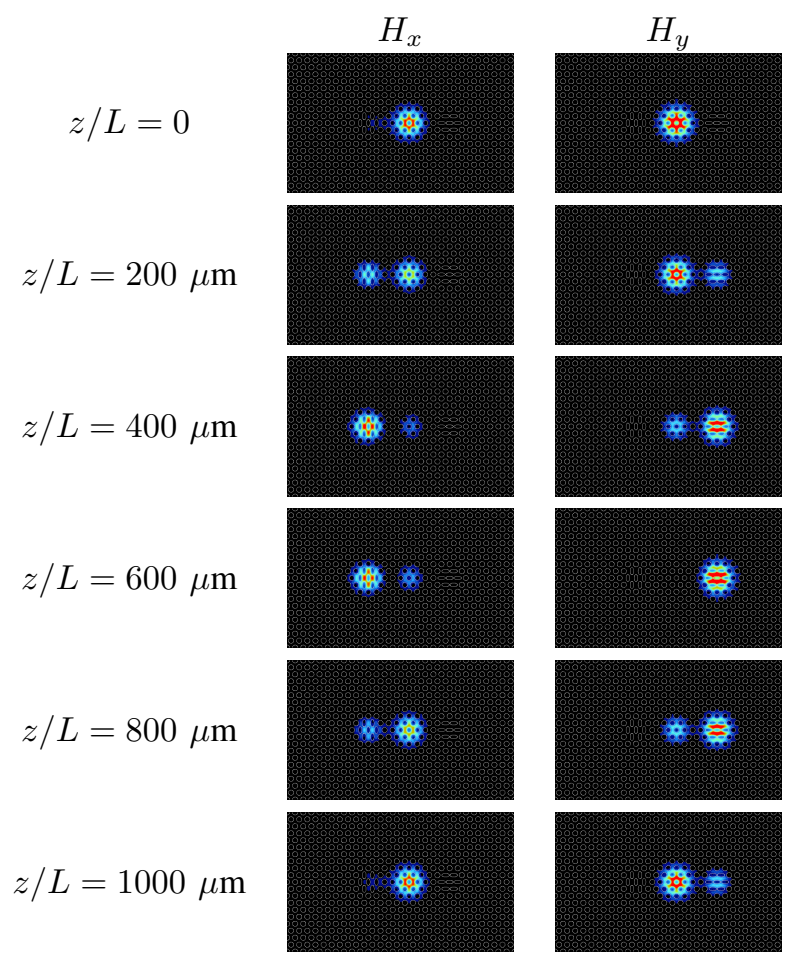

Fig. 5. Propagation behavior in the PS with the large hole EC-CHF designed by FSM.

arranged in the array of a hexagonal lattice with the hole pitch $\Lambda$ of $1.24 \mu \mathrm{m}$, the core region is formed by elliptical air holes with $d_{y 1}=0.9 \Lambda, d_{y 1} / d_{x 1}=2$, diameter of the circular hole in the cladding region is $d_{C}=0.65 \Lambda$, the refractive indices of silica and air holes are $n_{1}=1.45$ and $n_{2}=1$, respectively, and the operation wavelength is set to $\lambda=1.55 \mu \mathrm{m}$.

We employ the FV-FEM to estimate the modal effective index of the FSM for each waveguide. Fig. 3 shows the effective indices of the FSM in core region for the CC$\mathrm{CHF}$ and $x \mathrm{EC}-\mathrm{CHF}$ as a function of air filling fraction. The effective index of $y \mathrm{EC}-\mathrm{CHF}$ is illustrated by the dashed line. It can be seen that, the air hole sizes that meet the phase matching condition correspond to $d_{x 2}=0.6162 \Lambda$ for the CC-CHF, and $d_{x 3}=0.9222 \Lambda$ for the $x$ EC-CHF. However, numerical simulation demonstrates that in the isolated system, effective index of each EC-CHF with the hole size determined above cannot perfectly be matched. In the coupled system, the effective indices of even and odd modes and the estimated coupling lengths for the $x$ - and $y$-polarization are shown in Table I. There is a remarkable difference between $x$ - and $y$ polarization coupling lengths. The normalized power along propagation distance of $y$ - and $x$-polarized waves is illustrated in Fig. 4. Consequently, it can be seen that the incident light cannot completely split into two orthogonal polarization states. In particular, the maximum value of the normalized power in $y \mathrm{EC}-\mathrm{CHF}$ is 0.347 (coupling efficiency is about $69.30 \%$ ) at propagation distance of $482 \mu \mathrm{m}$. The propagation behavior in the PS with a device length of $1000 \mu \mathrm{m}$ can be seen in Fig. 5. In addition, the full-vector FE-BPM is applied to study the propagation along the $z$-direction. Here, the longitudinal step size is set to $1 \mu \mathrm{m}$, the analyzed region of $34.72 \times 24.8 \mu \mathrm{m}$, 


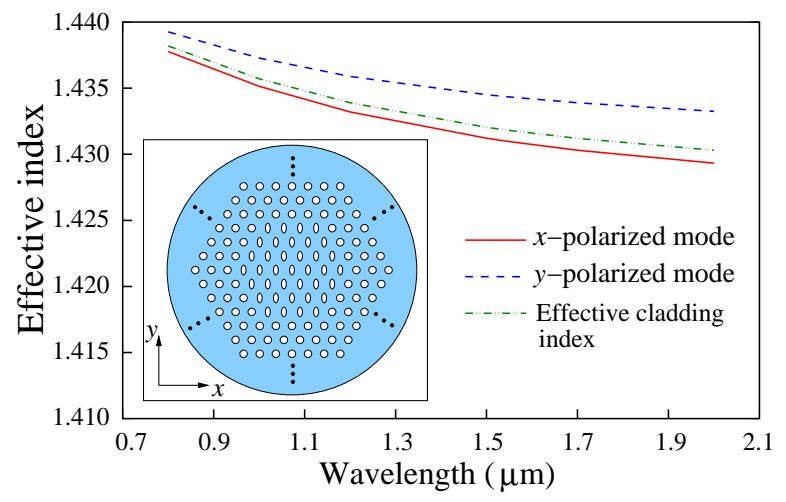

Fig. 6. Modal effective indices of $x$ - and $y$-polarized modes and cladding effective index of a small hole $y \mathrm{EC}-\mathrm{CHF}$ as a function of wavelength; the inset is a cross view of a 3 -ring core small hole $y$ EC-CHF.

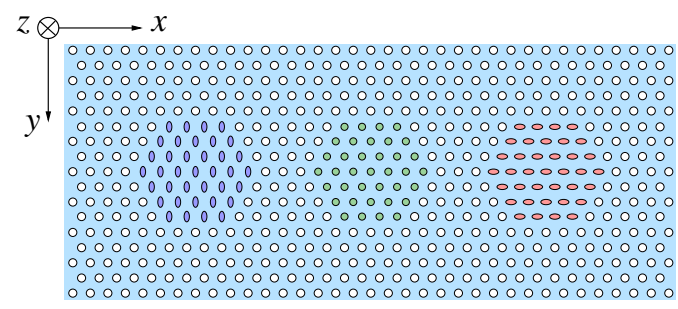

Fig. 7. Cross-section structure of the PS with the small hole EC-CHFs.

is discretized using curvilinear hybrid edge/nodal elements [19]. Adaptive mesh [20] is used and the total unknowns are 836,538 . For the $y$-polarized component, $H_{x}$ is the major field, and $H_{y}$ is the minor field. In the same way, for the $x$ polarized component, $H_{y}$ is the major field, and $H_{x}$ is the minor field. With the propagation of the light, we can observe that, $y$-polarized component of the incident light cannot totally couple into the $y \mathrm{EC}-\mathrm{CHF}$, and the remain part propagates in the CC-CHF due to the single-polarization property of ECCHF, and from this point, it also demonstrated that crosstalkfree polarization can be realized through our PS.

\section{B. Design of PS with small hole EC-CHFs}

After we designed the PS with the large hole EC-CHFs which have a strong optical confinement of the waveguide, in order to obtain the Gaussian like mode field distribution which can improve the connectivity between the EC-CHF and a standard single-mode fiber (SMF), we designed the PS with the small hole EC-CHFs. The parameters of $y$ EC-CHF are set as follows: $d_{y 1}=0.3 \Lambda$ for the major axis of elliptical holes, circular holes in cladding is $d_{c}=0.22 \Lambda$. The cross section of the small hole $y \mathrm{EC}-\mathrm{CHF}$ is shown in the inset of Fig. 6, it can be seen that the fiber includes three rings of elliptical holes in the core region to increase the optical confinement since the EC-CHF is weakly guiding waveguide with the small holes. The modal effective indices of the two orthogonal polarization and the cladding effective index of the $y \mathrm{EC}-\mathrm{CHF}$ as a function of wavelength are also shown in Fig. 6. From $800 \mathrm{~nm}$ to 2000 $\mathrm{nm}$, we can observed that only $y$-polarized mode can be guided well in the fiber core because its modal effective index is obviously higher than the cladding effective index, whereas the
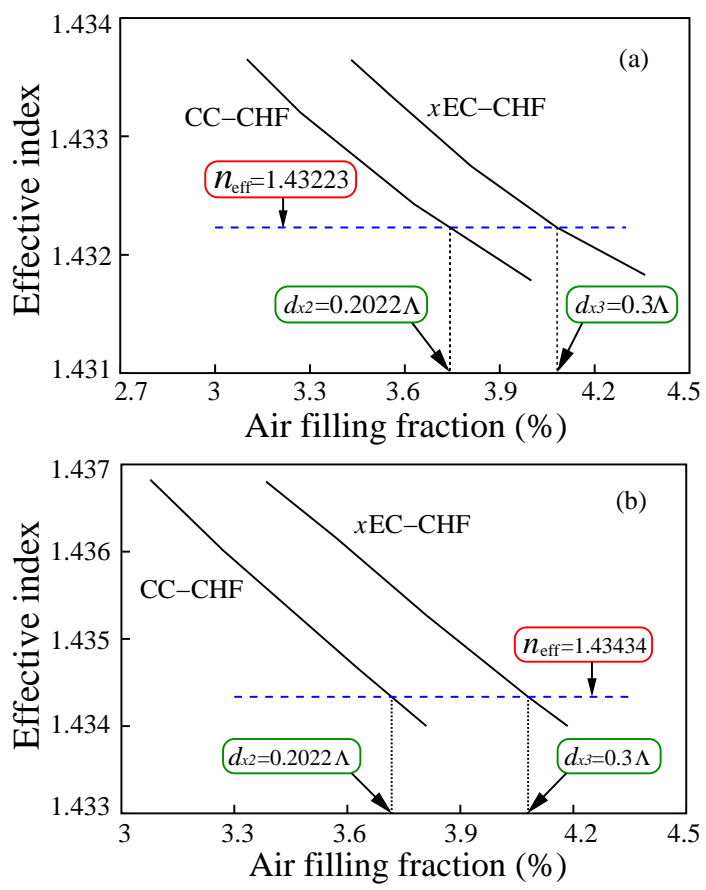

Fig. 8. Effective indices of (a) the CC-CHF and $x \mathrm{EC}-\mathrm{CHF}$, (b) the FSM in core region of the CC-CHF and $x \mathrm{EC}-\mathrm{CHF}$, as a function of the air filling fraction.

TABLE II

EFFECTIVE INDICES OF EVEN AND ODD MODES AND THE ESTIMATED COUPLING LENGTHS OF THE PS WITH THE SMALL HOLE EC-CHFS

\begin{tabular}{|c|c|c|c|}
\hline & \multicolumn{2}{|c|}{ Effective refractive index } & \\
\cline { 2 - 3 } & Even mode & Odd mode & $L_{c}[\mu \mathrm{m}]$ \\
\hline$x$-pol. & 1.432390 & 1.431992 & 1947 \\
\hline$y$-pol. & 1.432394 & 1.431993 & 1933 \\
\hline
\end{tabular}

$x$-polarized mode is lower than the cladding effective index. Fig. 7 shows the cross-sectional structure of the PS, and each core is separated from the adjacent cores by three air holes.

In such conditions, in order to match the phase condition of each EC-CHF, we use the two design method to determine the hole size in core region of CC-CHF and $x \mathrm{EC}-\mathrm{CHF}$ as same as the PS with the large hole EC-CHFs, that is the effective indices of waveguide which have high accuracy of coupling efficiency and only the FSMs of core region which are easy to design. Fig. 8 illustrates the vary of effective indices for the $\mathrm{CC}-\mathrm{CHF}$ and $x \mathrm{EC}-\mathrm{CHF}$ as a function of air filling fraction by the two design methods mentioned above. Here we note that the phase matched holes in the core region for the CC$\mathrm{CHF}$ and $x \mathrm{EC}-\mathrm{CHF}$ are the same size based on the two design methods, i.e. $d_{x 2}=0.2022 \Lambda$ for the CC-CHF and $d_{x 3}=0.3 \Lambda$ for the $x \mathrm{EC}-\mathrm{CHF}$. The effective indices of even and odd modes in coupled system and the estimated coupling lengths for $x$ and $y$-polarization have been calculated as shown in table II. The device length is set to $L=1940 \mu \mathrm{m}$.

The propagation behavior in the PS with device length of $1940 \mu \mathrm{m}$ is shown in Fig. 9. This time, the longitudinal step size is also set to $1 \mu \mathrm{m}$, however, the analyzed region is $62 \times 39.68 \mu \mathrm{m}$, and the total unknowns are $1,145,650$. A 45-degree linearly polarized light is launched into the CC- 


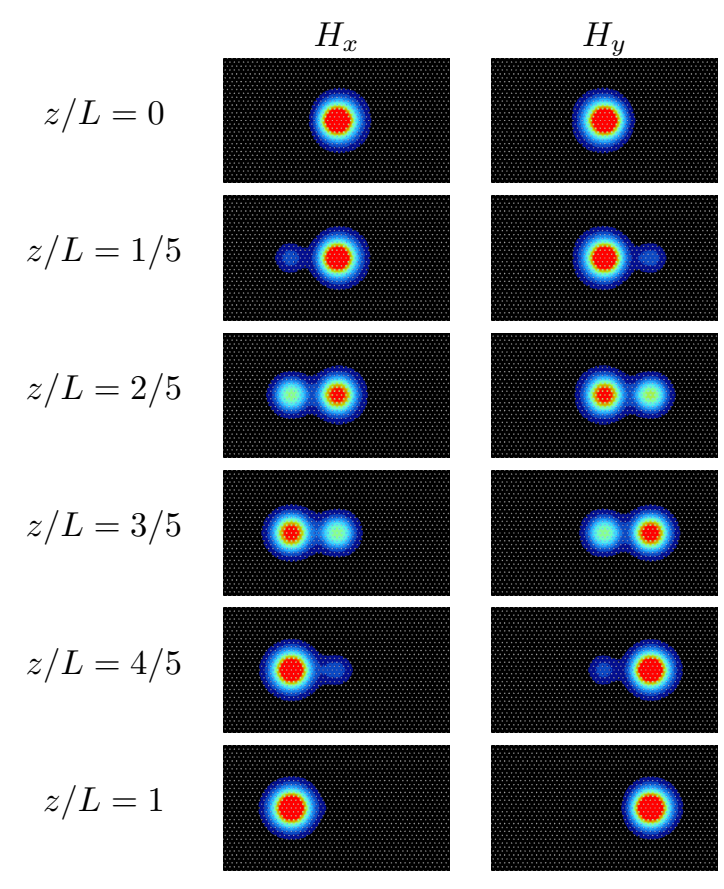

Fig. 9. Propagation behavior in the PS with the small hole EC-CHFs.

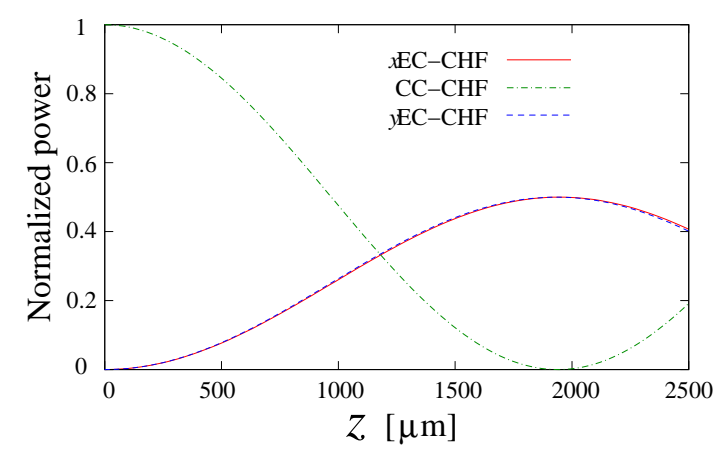

Fig. 10. Normalized power along the PS with the small hole EC-CHFs.

CHF, with the propagation of the light, it can be observed that the $x$-polarized component and $y$-polarized component couple only to the $x \mathrm{EC}-\mathrm{CHF}$ and $y \mathrm{EC}-\mathrm{CHF}$, respectively. We have also estimated the normalized power against propagation distance as illustrated in Fig. 10. The power of incident light in CC-CHF is almost 0 at $1940 \mu \mathrm{m}$, and $x$-polarized wave, $y$-polarized wave can be completely divided.

\section{Design accuracy of FSM method}

Compared with the coupling efficiencies of the PS by using the large hole EC-CHFs and small hole EC-CHFs based on FSM, we can observe that splitter with the large hole EC-CHFs cannot obtain a high coupling efficiency. As a consequence, we investigated the vary of coupling efficiency for $x$ - and $y$-polarization designed by FSM versus air filling fraction of core region, as shown in Fig. 11. Here, the ellipticity is considered as $d_{\text {major }} / d_{\text {minor }}=2$, and the distance between the two adjacent cores is fixed to two air holes. Reference to the original parameters of $y \mathrm{EC}-\mathrm{CHF}$ (the major axis of elliptical hole in core region is $0.9 \Lambda$, the diameter of circular

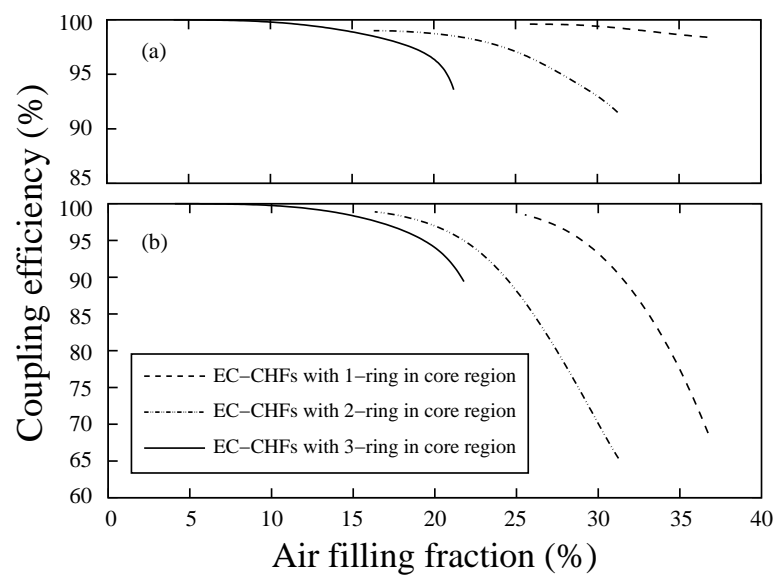

Fig. 11. Coupling efficiency of (a) $x$-polarized wave, (b) $y$-polarized wave for the PS designed by FSM as a function of air filling fraction.

hole in cladding is $0.65 \Lambda$ ), the circular hole size in cladding region varies as same ratio as the elliptical hole in core region to ensure the realization of single-polarization propagation of EC-CHF. In addition, as we know the EC-CHF with smaller holes leads to weaker light confinement, so we introduce a multi-ring structure in core region to enhance the light confinement of the waveguide when the hole sizes decrease. Fig. 11 shows that the coupling efficiencies of $x$ - and $y$ polarized waves decrease with the air filling fraction for each PS with different kinds of EC-CHFs. The normalized propagation constant $b$ of a waveguide is defined as follows:

$$
b=\frac{n_{\mathrm{eff}}^{2}-n_{2}^{2}}{n_{1}^{2}-n_{2}^{2}}
$$

where $n_{\text {eff }}$ is the effective index of the waveguide in isolated system, $n_{1}$ and $n_{2}$ represent the FSMs of core and cladding regions, respectively, and $b$ is a quantity between 0 and 1 . Small $b$ means the waveguide has a weak light confinement that can realize light coupling easily. On the other hand, a waveguide with a large $b$ has a strong light confinement and it is not easy to be coupled. $b$ of a waveguide increases with air filling fraction for each region of the EC-CHFs. For example, the PS with 1-ring core EC-CHFs (the range of the air filling fraction is from $25.51 \%$ to $36.73 \%$ ), it can be calculated that $b$ is 0.2725 and the coupling efficiency of $y$-polarization is $68.8 \%$ at the air filling fraction of $36.73 \%$. On the other hand, when the air filling fraction is $25.51 \%, b$ is 0.1185 and the coupling efficiency of $y$-polarization is $98.5 \%$. Weakly light confinement fibers have a large insertion loss of input and output fiber even though it can realize the light coupling easily. Therefore, the 2-ring core structure is generally used at the air filling fraction of $25.51 \%$, although the coupling efficiency of $y$-polarization decreases to $87.4 \%$. In the actual fabrication, according to the normalized propagation constant of needs, we determine the rings in the core region of the reference waveguide (such as the $y$ EC-CHF in this paper) and the distance between the two adjacent cores, then we can choose the high design accuracy of FSM method to design the PS effectively. 


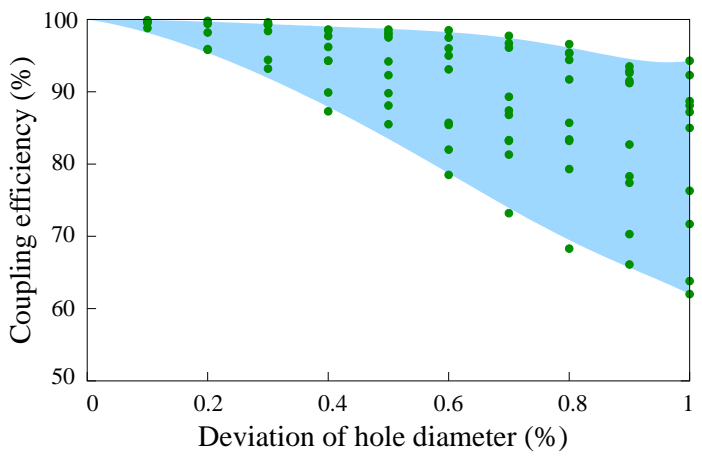

Fig. 12. Coupling efficiencies of the PS with the large hole EC-CHFs against the random deviation of all the air holes.

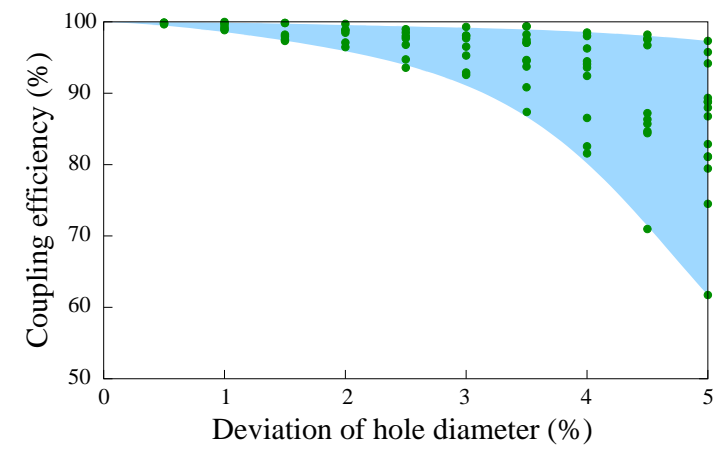

Fig. 13. Coupling efficiencies of the PS with the small hole EC-CHFs against the random deviation of all the air holes.

\section{TOLERANCE AND WAVELENGTH DEPENDENCE OF PS}

Considering the current fabrication technology of PCF, the biggest problem in the fabrication process of EC-CHFs is the deviation of the circular or elliptical air holes away from the initial parameter values. So we discussed the effect of the deviation of the geometric parameters designed with the large and small hole EC-CHFs. Since the air holes deviate irregularly in fabrication, we investigated the coupling efficiency of the PS against all air holes varies randomly from the initial parameters. Reference to [18], the parameters of each large hole EC-CHF are set to be as follows which can split light into two orthogonal states completely: $d_{y 1}=0.9 \Lambda$ for the $y \mathrm{EC}$ CHF, $d_{x 2}=0.6126 \Lambda$ for the CC-CHF, $d_{x 3}=0.9174 \Lambda$ for the $x$ EC-CHF, the ellipticity is $d_{\text {major }} / d_{\text {minor }}=2, d_{C}=0.65 \Lambda$ for the holes in cladding, each core is separated from the adjacent cores by two air holes, and the device length is fixed to $630 \mu \mathrm{m}$. Fig. 12 shows the coupling efficiency of the PS with the large hole EC-CHFs against the random deviation of all the air holes. It can be observed that the coupling efficiency is almost $90 \%$ when the deviation is smaller than $0.4 \%$ of their initial parameters, and the coupling efficiency is better than $60 \%$ when the deviation is smaller than $1 \%$ of the initial parameters. On the other hand, Fig. 13 shows the coupling efficiency of the PS with the small hole EC-CHFs designed in section 3 against the random deviation of all the air holes. Compared with the condition in Fig. 12, the coupling efficiency is better than $90 \%$ when the deviation is smaller than $3 \%$ of their initial parameters, and the coupling efficiency is better than $60 \%$ when the deviation is smaller than $5 \%$ of the
TABLE III

PS WITH LARGE EC-CHF: COUPLING EFFICIENCY VERSUS THE DEVIATION FOR DIFFERENT PARTS OF THE DEVICE PARAMETERS AT EACH DEVIATION LEVEL

\begin{tabular}{|c|c|c|c|c|}
\hline \multirow{2}{*}{$\begin{array}{c}\text { Deviation } \\
\text { level }\end{array}$} & \multicolumn{3}{|c|}{ Core region } & Cladding \\
\cline { 2 - 4 } & $x$ EC-CHF & CC-CHF & $y$ EC-CHF & region \\
\hline$-0.3 \%$ & $88.9 \%$ & $85.4 \%$ & $87.6 \%$ & $99.8 \%$ \\
\hline$-0.2 \%$ & $94.5 \%$ & $92.6 \%$ & $94.3 \%$ & $99.7 \%$ \\
\hline$-0.1 \%$ & $97.9 \%$ & $97.2 \%$ & $98.4 \%$ & $99.3 \%$ \\
\hline 0 & $99.9 \%$ & $99.9 \%$ & $99.9 \%$ & $99.9 \%$ \\
\hline $0.1 \%$ & $97.8 \%$ & $98.0 \%$ & $98.2 \%$ & $98.6 \%$ \\
\hline $0.2 \%$ & $94.3 \%$ & $94.2 \%$ & $94.0 \%$ & $98.0 \%$ \\
\hline $0.3 \%$ & $88.9 \%$ & $88.0 \%$ & $87.6 \%$ & $97.3 \%$ \\
\hline
\end{tabular}

TABLE IV

PS WITH SMALL EC-CHF: COUPLING EFFICIENCY VERSUS THE DEVIATION FOR DIFFERENT PARTS OF THE DEVICE PARAMETERS AT EACH DEVIATION LEVEL

\begin{tabular}{|c|c|c|c|c|}
\hline \multirow{2}{*}{$\begin{array}{c}\text { Deviation } \\
\text { level }\end{array}$} & \multicolumn{3}{|c|}{ Core region } & Cladding \\
\cline { 2 - 5 } & $x$ EC-CHF & CC-CHF & $y$ EC-CHF & region \\
\hline$-1 \%$ & $86.1 \%$ & $86.0 \%$ & $86.3 \%$ & $99.8 \%$ \\
\hline$-0.66 \%$ & $94.0 \%$ & $93.6 \%$ & $94.1 \%$ & $99.9 \%$ \\
\hline$-0.33 \%$ & $98.6 \%$ & $98.3 \%$ & $98.6 \%$ & $99.9 \%$ \\
\hline 0 & $100 \%$ & $100 \%$ & $100 \%$ & $100 \%$ \\
\hline $0.33 \%$ & $98.3 \%$ & $98.7 \%$ & $98.4 \%$ & $99.9 \%$ \\
\hline $0.66 \%$ & $94.0 \%$ & $94.9 \%$ & $94.2 \%$ & $99.9 \%$ \\
\hline $1 \%$ & $87.7 \%$ & $88.9 \%$ & $87.8 \%$ & $99.8 \%$ \\
\hline
\end{tabular}

initial parameters. The PS with the small hole EC-CHFs has a higher tolerance than the splitter with the large hole ECCHFs because it has more elliptical air holes in core region which lead to reducing the average of deviation and decrease the impact to effective index of the waveguide.

Moreover, we have also examined the coupling efficiency versus the deviation for the respective hole sizes of each region, as shown in Table III and Table IV. Here, all the hole sizes of the deviation part are varied with the same extent, such as if only the holes in $x \mathrm{EC}-\mathrm{CHF}$ core region are expanded $1 \%$, the coupling efficiency of the PS with small hole ECCHFs will decrease to $87.7 \%$. Compared with the random deviation of all the holes mentioned above, the deviation for one part of the device with same extent leads to worse coupling efficiency. It can be observed that the coupling efficiency with deviation in the core regions is much worse than the varies in the cladding, that is because in a EC-CHF, the effective index is sensitive to the hole sizes in the core region. So if we design this type of PS using other kinds of single-polarized PCFs [3][10] which have less holes or no holes in the core region, it may be possible to obtain a device with larger tolerance.

In order to achieve the wide-band transmission, we have also investigated the wavelength dependence of the PS with the large and small hole EC-CHFs, respectively, as shown in Fig. 14. The proposed PS with the large hole EC-CHFs has a coupling efficiency better than $20 \mathrm{~dB}$ at $1.55 \mu \mathrm{m}$ and a bandwidth of $50 \mathrm{~nm}$ from 1.52 to $1.57 \mu \mathrm{m}$, the PS with the small hole EC-CHFs exhibits a wider bandwidth of 160 $\mathrm{nm}$ from 1.47 to $1.63 \mu \mathrm{m}$. That is because the EC-CHF with 


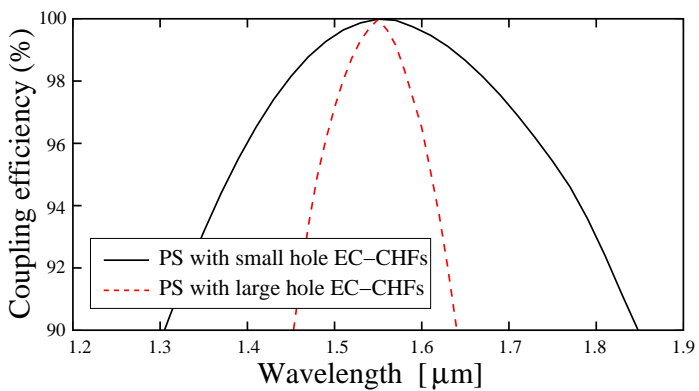

Fig. 14. Coupling efficiency of the PS versus the light wavelength.

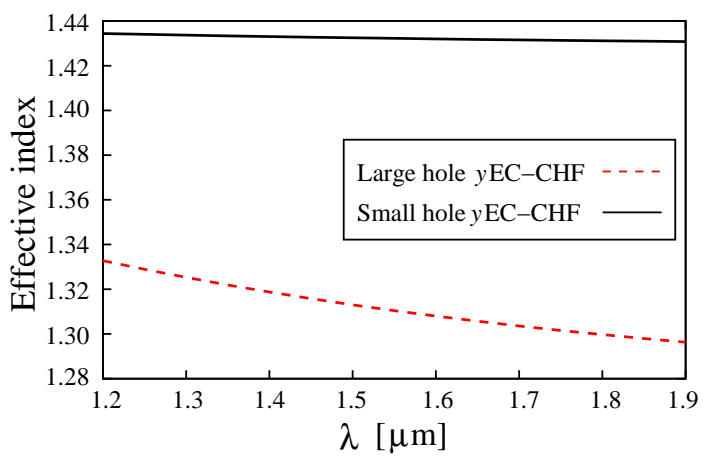

Fig. 15. Effective index of the $y$ EC-CHF versus wavelength.

small holes is a weakly guiding, and the effective index of the waveguide varies slightly versus wavelength, as shown in Fig. 15. So the proposed small hole PS has a wider bandwidth.

\section{CONCLUSiON}

In this paper, we designed a novel PS using the singlepolarized EC-CHFs which can divide the incident light into two orthogonal states completely without any cross-talk. FSM of each waveguide is used to design the PS efficiently, we discussed the design accuracy of FSM method in detail. The tolerance and wavelength dependence of the PS with the large hole EC-CHFs and small hole EC-CHFs have also been investigated.

\section{REFERENCES}

[1] J. C. Knight, T. A. Birks, P. St. J. Russell, and D. M. Atkin, "All-silica single-mode optical fiber with photonic crystal cladding," Opt. Lett., Vol. 21, Issue 19, pp. 1547-1549, Oct. 1996.

[2] P. St. J. Russell, "Photonic-crystal fibers," J. Ligthwave Technol., Vol. 24, No. 12, pp. 4729-4749, Dec. 2006.

[3] K. Satioh and M. Koshiba, "Single-polarization single-mode photonic crystal fibers," IEEE Photon. Technol. Lett., Vol. 15, No. 10, pp. 13841386, Oct. 2003.

[4] J. R. Folkenberg, M. D. Nielsen, and C. Jakobsen, "Broadband singlepolarization photonic crystal fiber," Opt. Lett., Vol. 30, No. 12, pp. 14461448, June 2005.

[5] F. Zhang, M. Zhang, X. Liu, and P. Ye, "Design of wideband singlepolarization single-mode photonic crystal fiber," J. Ligthwave Technol., Vol. 25, No. 5, pp. 1184-1189, May 2007.

[6] M. Eguchi and Y. Tsuji, "Single-mode single-polarization holey fiber using anisotropic fundamental space-filling mode," Opt. Lett., Vol. 32, No. 15, pp. 2112-2114, Aug. 2007.

[7] M. Eguchi and Y. Tsuji, "Design of single-polarization elliptical-hole core circular-hole holey fibers with zero dispersion at $1.55 \mu \mathrm{m}$," J. Opt. Soc. Am. B, Vol. 25, No. 10, pp. 1690-1701, Oct. 2008.
[8] D. Hu, P. Shum, C. Lu, X. Yu, G. Wang, and G. Ren, "Holey fiber design for single-polarization single-mode guidance," Appl. Opt., Vol. 48, No. 20, pp. 4038-4043, July 2009.

[9] R. Goto, S. D. Jackson, and K. Takenaga, "Single-polarization operation in birefringent all-solid hybrid microstructured fiber with additional stress applying parts," Opt. Lett., Vol. 34, No. 20, pp. 3119-3121, Oct. 2009.

[10] M. Eguchi, and Y. Tsuji, "Single-polarization elliptical-hole lattice core photonic-bandgap fiber," J. Lightwave Technol., Vol. 31, No. 1, pp. 177 182, Jan. 2013.

[11] L. Zhang and C. Yang, "Polarization splitter based on photonic crystal fibers," Opt. Express, Vol. 11, No. 9, pp. 1015-1020, May 2003.

[12] K. Saitoh, Y. Sato and M. Koshiba, "Polarization splitter in three-core photonic crystal fibers," Opt. Express, Vol. 12, No. 17, pp. 3940-3946, Aug. 2004.

[13] L. Zhang and C. Yang, "A novel polarization splitter based on the photonic crystal fiber with nonidentical dual cores," IEEE Photon. Technol. Lett., Vol. 16, No. 7, July 2004.

[14] L. Zhang and C. Yang, "Polarization-dependent coupling in twin-core photonic crystal fibers," J. Lightwave Technol., Vol. 22, No. 5, pp. 13671373, May 2004.

[15] L. Zhang, C. Yang, C. Yu, T. Luo and A. E. Willner, "PCF-based polarization splitters with simplified structures," J. Lightwave Technol., Vol. 23, No. 11, pp. 3558-3565, Nov. 2005.

[16] M. Chen, B. Sun, Y. Zhang and X. Fu, "Design of broadband polarization splitter based on partial coupling in square-lattice phtotnic-crystal fiber," Appl. Opt., Vol. 49, No. 16, pp. 3042-3048, June 2010.

[17] W. Lu, S. Lou, X. Wang, L. Wang and R. Feng, "Ultrabroadband polarization splitter based on three-core photonic crystal fibers," Appl. Opt., Vol. 52, No. 3, pp. 449-455, Jan. 2013.

[18] Z. Zhang, Y. Tsuji and M. Eguchi, "Design of polarization splitter with single-polarized elliptical-hole core circular-hole holey fibers," IEEE Photon. Technol. Lett., Vol. 26, No. 6, pp. 541-543, Mar. 2014.

[19] M. Koshiba and Y. Tsuji, "Curvilinear hybrid edge/nodal elements with triangular shape for guidedwave problems," J. Ligthwave Technol., Vol. 18, No. 5, pp. 737-743, May 2000.

[20] Y. Tsuji and M. Koshiba, "Adaptive mesh generation for full-vectorial guided-mode and beam propagation solutions," J. Sel. Topics Quantum Electron., Vol. 6, No. 1, pp. 163-169, Jan. 2000.

[21] M. Eguchi and Y. Tsuji, "Influence of reflected radiation waves caused by large mode field and large refractive index mismatches on splice loss evaluation between elliptical-hole lattice core holey fibers and conventional fibers," J. Opt. Soc. Am. B, Vol. 30, No. 2, pp. 410-420, Feb. 2013.

Zejun Zhang (S' 14) was born in Jiaozuo, Henan, China, on April 3, 1989. He received the B.S. degree in department of electrical engineering from Xuchang University, Xuchang, Henan, China, in 2011, and he received the M.S. degree in division of information and electronic engineering from Muroran Institute of Technology, Muroran, Japan, in 2014.

Yasuhide Tsuji (M’ 97) was born in Takikawa, Japan, on December 31, 1967. He received the B.S., M.S., and Ph.D. degrees in electronic engineering from Hokkaido University, Sapporo, Japan, in 1991, 1993, and 1996, respectively.

$\mathrm{He}$ is a Professor of Division of Information and Electronic Engineering at Muroran Institute of Technology, Muroran, Japan. He has been engaged in research on optical wave electronics.

Masashi Eguchi (M'93) was born in Sapporo, Japan, on August 9, 1962. He received the B.S. degree in electronic engineering from Kitami Institute of Technology, Kitami, in 1985 and the M.S. and Ph.D degrees in electronic engineering from Hokkaido University, Sapporo, Japan, in 1987, 1991, respectively.

$\mathrm{He}$ is an Associate Professor of the Faculty of Photonics Science, Chitose Institute of Science and Technology, Chitose, Japan. He has been engaged in research on various optical fibers, dielectric waveguides, optical solitons, and applications of finite element method and other electromagnetic wave analysis methods. 